\title{
MicroRNAs regulate gene plasticity during cold shock in zebrafish larvae
}

\author{
I-Chen Hung ${ }^{1 \dagger}$, Yu-Chuan Hsiao ${ }^{1 \dagger}$, H. Sunny Sunn ${ }^{2,3}$, Tsung-Ming Chen ${ }^{4^{*}}$ and Shyh-Jye Lee ${ }^{1,5,6,7^{*}}$ (D)
}

\begin{abstract}
Background: MicroRNAs (miRNAs) are critical regulators responding to acute environmental stresses in both plants and animals. By modulating gene expression, miRNAs either restore or reconstitute a new expression program to enhance cell tolerance to stresses. Cold shock is one of the stresses that can induce acute physiological responses and transcriptional changes in aquatic creatures. Previous genomic studies have revealed many cold-affected genes in fish larvae and adults, however, the role of miRNAs in acute cold response is still ambiguous. To elucidate the regulatory roles of miRNAs in the cold-inducible responses, we performed small RNA-seq and RNA-seq analyses and found potential cold regulatory miRNAs and genes. We further investigated their interactions and involvements in cold tolerance.

Results: Small RNA-seq and RNA-seq identified 29 up-/26 down-regulated miRNAs and 908 up-/468 downregulated genes, respectively, in responding to cold shock for $4 \mathrm{~h}$ at $18^{\circ} \mathrm{C}$. miRNA and transcriptomic analyses showed these miRNAs and mRNAs are involved in similar biological processes and pathways. Gene ontology enrichment analyses revealed the cold-induced genes were enriched in pathways, including melanogenesis, GnRH pathway, circadian rhythm, etc. We were particularly interested in the changes in circadian clock genes that affect daily metabolism. The enrichment of circadian clock genes was also observed in previous fish cold acclimation studies, but have not been characterized. To characterize the functional roles of circadian clock genes in cold tolerance, we individually overexpressed selected clock genes in zebrafish larvae and found one of the core clock genes per2 resulted in better recovery from cold shock. In addition, we validated the interaction of per2 with its associate miRNA, dre-mir29b, which is also cold-inducible. It suggests the transcription of per2 can be modulated by miRNA upon cold shock.
\end{abstract}

Conclusions: Collectively, our observations suggest that miRNAs are fine turners for regulating genomic plasticity against cold shock. We further showed that the fine tuning of core clock gene per2 via its associated miRNA, dre-mir-29b, can enhance the cold tolerance of zebrafish larvae.

Keywords: Deep sequencing, miRNA, Clock genes, Zebrafish, Embryonic development, Cold stress

\section{Background}

The survival of aquatic fish is constantly challenged by the changes in environmental factors, such as salinity, oxygen concentration, $\mathrm{pH}$, osmotic pressure and temperature. The change in water temperature has a dramatic effect on physiology and behaviors of aquatic animals that it has been shown to be an "abiotic master factor" for fish $[1,2]$. Cold shock is characterized as an acute reduction in

\footnotetext{
*Correspondence: tmtonychen@webmail.nkmu.edu.tw; jefflee@ntu.edu.tw ${ }^{\dagger}$ Equal contributors

${ }^{4}$ Department and Graduate Institute of Aquaculture, National Kaohsiung Marine University, Kaohsiung 81157, Taiwan

'Department of Life Science, National Taiwan University, Taipei 10617, Taiwan

Full list of author information is available at the end of the article
}

ambient temperature that may cause rapid falling in body temperature and a cascade of physiological and behavioral responses [2]. The cold shock stress response in fish can be broadly grouped into three categories; primary, secondary, and tertiary responses [2-4]. The primary responses of cold shock rapidly change the neuroendocrine pathways, such as catecholamines and corticosteroids [5-7]. It is initiated by the central nervous system sensing low temperatures that stimulates the chromaffin cells to release catecholamines. The hypothalamus-pituitary-interrenal axis is then activated to secret corticotrophin-releasing hormone to stimulate adrenocorticotrophic hormone and subsequent release of cortisol $[4,8,9]$. Secondary response 
can be elicited by the primary response. The stress hormones and cortisol interact to induce glucose production in fish via glycogenolysis and glucogenesis $[9,10]$ that results in changes of protein expression, molecular responses, osmoregulatory, and immune systems $[2,11]$. The tertiary responses are the global effects of cold shock to impair development rate, respiratory function, swimming behavior, and mortality [2].

Expressions of numerous genes are regulated by stresses in fish. Transcriptome analysis has been used in the discovery of many stress-inducible genes in cold acclimated [12-14] or cold-shocked fishes [15, 16]. Those genes belong to a variety of biological processes, including chaperones, transcription factors, signal transduction pathways, metabolism, responses to stress or ion transporters like kir2.2 channel in rainbow trout [17], heat shock protein 70 in silver sea bream [18] and glycerol-3-phosphate dehydrogenase in rainbow smelt [19]. However, studies on non-model fishes have been difficult due to the lack of whole genome sequences and inability for genome manipulation. Zebrafish is a favorable model fish due to the availability of whole gene sequences and accessibility of genome manipulation. They can also be used for genomic research using high-throughput RNA-seq and small RNA-seq techniques. Moreover, zebrafish is a tropical animal acclimated to extreme temperature changes ranging from 6.2 to $39.2{ }^{\circ} \mathrm{C}$ in certain seasons [20]. This makes it an ideal model to investigate the regulatory mechanisms of temperaturedependent responses.

MicroRNAs (miRNAs) are short non-coding RNAs of around 22 nucleotides, which can modulate the stability and silence mRNAs [21]. A miRNA typically targets multiple mRNAs [22], which makes it a potential finetuner for different situations. Studies indicate that miRNAs play a key role in gene regulation in stress responses like oxidative reaction [23], temperature [24] or DNA damage stresses [25] in different organisms [26, 27]. However, relatively less is known about the functions of miRNA in aquatic species. miR-8 and miR-429 have been reported to be involved in the osmotic stress response in zebrafish $[28,29]$. In contrast, high-throughput miRNAtomes analysis revealed no significant impact by miRNA-mediated gene regulation in the cold - acclimated adult zebrafish brains [30]. This result is puzzling, but we reasoned that the cold-induced responses might be different in organisms or tissues used. For example, fish larvae are more voluntary to environmental stresses like acute cold shock, but their responses to the changes in miRNA and transcriptomic profiles during acute cold shock remains unexplored.

Yang et al. claimed that miRNAs may have a minor role in regulating transcriptome plasticity in cold acclimated zebrafish brains based on the poor correlations between cold-induced transcriptomic and miRNAtome profiles [30]. This is in clear contrast to our previous understanding observed in other organisms [31, 32]. The discrepancy may be due to differences in organisms, tissue analyzed and experimental conditions. To further clarify this issue, we aimed to investigate the correlation between miRNAtome and transcriptome under cold shock in zebrafish larvae and to resolve possible colddependent regulatory mechanisms of selected miRNAs and their target genes. We discovered that similar biological processes and pathways were affected by cold in both miRNAtome and transcriptome analyses. In particular, we found notable correlations between circadian rhythm-related genes (known as circadian clock genes) [33] and their associate miRNAs. Core clock gene per2 plays a major role in regulating circadian rhythm and daily metabolism [34]; we found that dre-mir-29b (dre denotes Danio rerio) can target per2 in zebrafish larvae to possibly regulate cold shock response via modulating the expression of per2. More importantly, we demonstrated that the overexpression of per 2 in zebrafish larvae can increase their cold tolerance. These results indicate a pivotal role of miRNA in regulating gene plasticity to counter cold stress.

\section{Methods}

\section{Fish maintenance}

Zebrafish, Danio rerio, wild-type AB strain, were maintained under a $14 \mathrm{~h} \mathrm{light} / 10 \mathrm{~h}$ dark cycle at $28.5^{\circ} \mathrm{C}$. Embryos were collected by natural spawning and incubated in $0.3 \times$ Danieau's buffer (1× Danieau's buffer: $58 \mathrm{mM}$ $\mathrm{NaCl}, 0.7 \mathrm{mM} \mathrm{KCl}, 0.4 \mathrm{mM} \mathrm{MgSO}_{4}, 0.6 \mathrm{mM} \mathrm{Ca}\left(\mathrm{NO}_{3}\right)_{2}$, and 5.0 mM HEPES in double - distilled water, $\mathrm{pH}$ adjusted at 7.6) until examination or fixation.

\section{Cold shock and embryo collection}

For cold shock experiment, 50 larvae were cultured at $28.5{ }^{\circ} \mathrm{C}$ until $96 \mathrm{~h}$ post fertilization (hpf), transferred into a $250 \mathrm{ml}$ beaker containing $100 \mathrm{ml}$ pre-chilled $0.3 \times$ Danieau's buffer, and immediately incubated at designated temperatures until examination. The 96-hpf embryos before cold shock were collected as the control group. Embryos incubated at $28.5^{\circ} \mathrm{C}$ or $18{ }^{\circ} \mathrm{C}$ for 4 more hours were collected as the normal or cold shock group, respectively. Two different batches of samples were collected for further next generation sequencing (NGS) analysis.

\section{miRNA library construction and sequencing}

RNA samples were collected from the control, normal and cold shock groups for cDNA library construction. RNAs of desired time points were isolated by using the TRIzol $^{\circ}$ Reagent (Life technologies, Carlsbad, CA), and then the amount and quality of RNAs were assessed 
using the NanoDrop ${ }^{\circ}$ ND-1000 Spectrophotometer (ThermoFisher, Waltham, MA), Bioanalyzer 2100, RNA 6000 Nano Kit and Small RNA Chip Kit (Agilent Technology, Santa Clara, CA). For miRNA library construction and sequencing, miRNAs were enriched using the PureLink $^{\text {TM }}$ miRNA Isolation Kit (Invitrogen, Carlsbad, $\mathrm{CA}$ ), and then adaptors were hybridized and ligated to the $5^{\prime}$ and $3^{\prime}$ ends of these RNAs using the SOLiD ${ }^{\mathrm{ru}}$ Total RNA-Seq Kit (Applied Biosystems Inc., ABI, Carlsbad, CA). The ligated RNAs were used as templates for cDNA synthesis followed by cDNAs purification using the MinElute ${ }^{\circ}$ PCR Purification Kit (QIAGENE, Valencia, CA). The cDNAs at $120-130$ base pair (bp) were selected (Invitrogen, Novex ${ }^{\bullet}$ pre-cast gel products), amplified (ABI, SOLiD ${ }^{\text {tw }}$ Total RNA-Seq Kit), purified (Invitrogen, PureLink ${ }^{\text {Th }}$ PCR Micro Kit), and proceeded with SOLiD $^{\text {ma }}$ System template bead preparation (ABI, SOLiD $^{\text {tu }}$ RNA Barcoding Kit). The libraries were sequenced with a read length of $35 \mathrm{bp}$ using the SOLiD $5500 \mathrm{xl}$ sequencer.

\section{CDNA library construction and sequencing}

RNA samples were collected as described for cDNA library construction. RNAs of desired time points were isolated as described and used RiboMinus ${ }^{\mathrm{mm}}$ (Invitrogen) to remove ribosomal RNAs. For the preparation of cDNA libraries, the RNAs were fragmented using the RNAase III by using the Library Builder ${ }^{\mathrm{Tm}}$ Whole Transcriptome Core Kit (ABI) and cleaned up by using the Fragmented RNA Concentrator Module Kit (ABI). To amplify and purify the cDNA libraries we used the Library Builder ${ }^{\mathrm{Tm}}$ Whole Transcriptome Core Kit (ABI). The cDNA libraries with a size between 200 and $300 \mathrm{bp}$ were proceeded with the SOLiD ${ }^{\text {mi }}$ System template bead preparation using the EZ Bead ${ }^{\text {Tw }}$ System (ABI). The libraries were sequenced with read length of $75 \mathrm{bp}$ using the SOLiD $5500 \mathrm{xl}$ sequencer.

Identification of differential expressed genes and miRNAs Raw reads from the SOLiD $5500 \mathrm{xl}$ sequencing were analyzed using the LifeScope ${ }^{\mathrm{Tw}}$ Genomic Analysis Software. Those reads were filtered for high quality, adaptor selfligation, transfer RNAs and ribosomal RNAs (http:// rfam.sanger.ac.uk/). These filtering steps resulted in high quality filtered reads representing zebrafish RNA sequences. The filtered reads were mapped to the zebrafish genome sequences (Zv9, http://www.sanger.ac.uk/resources/downloads/zebrafish/) and annotated either the miRBase v18 (http://www.mirbase.org/) for miRNAs or the zebrafish genome annotation file $(\mathrm{Zv} 9 /$ danRer7 from UCSC) for transcripts. Annotated reads were further normalized to the reads per kilo base per million (RPKM). The differentially expressed miRNA and genes were identified and compared using the Partek Genomics Suite (http://www.partek.com/).

\section{Quantitative PCR analysis}

The total RNAs were isolated as described and genomic DNAs were removed by using the DNA-Free kit (Life technologies). One microgram of total RNAs was reversely transcribed to miRNAs and cDNAs by the miScript II RT Kit (QIAGENE). The quantitative polymerase chain reaction (qPCR) was performed using the miScript SYBR Green PCR Kit (QIAGENE) for miRNA quantification. The zebrafish U6 ( $5^{\prime}$-ACTAAAATTGGAACGA TACAGAGA-3') and efl $\alpha$ served as the internal control for miRNA and mRNA, respectively. $\mathrm{qPCR}$ analyses were performed by using the $\mathrm{iQ}^{\text {tw }} \mathrm{SYBR}^{\circ}$ Green Supermix in a CFX96 station (Bio-Rad, Hercules, California). Primers used are listed in the Additional file 1.

\section{Gene cloning}

Coding sequences of period 2 (per2, ENSDARG 00000034503), aryl hydrocarbon receptor nuclear translocator-like 1a (arntl1a, ENSDARG00000006791), basic helix-loop-helix family, member e41 (bhlhe41, ENSDARG00000041691) were amplified from the zebrafish total cDNAs by reverse transcriptase PCR (RTPCR). Total RNAs of zebrafish embryos were isolated as described and reversely transcribed by the MMLV reverse transcriptase (Promega, Fitchburg, Wisconsin). Primers were designed according to the respective reference RNA sequence. The amplicons were sub-cloned into a pT2MUAS vector for Tol2-mediated transgenesis [35]. The vector also contains a viral $2 \mathrm{~A}$ peptide sequence for the separation of the expressed protein from a nuclear reporter H2AmCherry used for founder fish screening.

\section{Ectopic expression and analysis of circadian clock genes in zebrafish larvae}

One-cell stage zebrafish embryos were injected with 25 pg Gal4 mRNAs, 25 pg Toll 2 mRNA and 50 pg plasmids and incubated under a $14 \mathrm{~h} / 10 \mathrm{~h}$ dark/light cycle until examination. Embryos with H2AmCherry expression were selected for further analysis.

\section{Swimming behavior analysis}

Five days post fertilization (dpf) larvae expressing with or without per2, arntl1a or bhlhe41 were individually distributed into each well of a 24-well plate with $1.5 \mathrm{ml}$ of $0.3 \times$ Danieau's buffer and incubated for $4 \mathrm{~h}$ at 28.5 or $18{ }^{\circ} \mathrm{C}$. Following incubation, the larvae were transferred to $28.5{ }^{\circ} \mathrm{C}$ and videotaped in a controlled light-on DanioVision observation chamber for $30 \mathrm{~min}$. The tracking of swimming for each larva was analyzed during 0 10 min, 10-20 mins and 20-30 mins using the 
EthoVision XT 8.5 software (Noldus Information Technology, Wageningen, The Netherlands).

\section{Measurement of glucose concentration}

Control or transient expressing embryos were cultured and cold shocked as described. Ten larvae for each treatment were collected at 0,2 , and $4 \mathrm{~h}$ after cold-shock, lysed in $200 \mu \mathrm{l}$ RIPA buffer (ThermoFisher Scientific, Waltham, MA), and the glucose concentration was measured by using the Amplite ${ }^{\mathrm{TM}}$ Fluorimetric Glucose Quantitation Kit (AAT-bioquest, Sunnyvale, CA).

\section{Reporter assay of dre-mir-29b}

The 3' untranslated region (UTR) of per2 containing dre-mir-29b target site was amplified from the cDNA library by the forward $(\mathrm{F})$ and reverse $(\mathrm{R})$ primers of the following sequences. F: Bgll2-Stop-per2 ATCGAGATCT TAAAATTCCTTTCGCATTCACAA and R: SacI-per2ATCGGAGCTCAGTCTGTGAGATCAGTTAAACCA. The amplicons were cloned into the pEGFP-C1 vector by selected restriction enzyme site SacI and Bgll2. Plasmids were mixed with or without dre-mir-29b morpholino (5'ACACTGATTTCAAATGGTGCTAGAT3'). The plasmid mix was injected into 1-cell zebrafish embryos and observed under epifluorescent microscopy at $10 \mathrm{hpf}$.

\section{Statistical analysis}

All experimental values are presented as mean \pm standard error and were analyzed by unpaired-sample Student's $t$-test and one-way ANOVA.

\section{Results}

\section{Cold-induced responses in zebrafish larvae}

To investigate cold-induced responses in zebrafish, $96 \mathrm{hpf}$ larvae were exposed to decreasing temperatures to monitor their mobility and mortality for $10 \mathrm{~h}$ (Table 1 ). Fish were mostly normal from 28.5 to $20{ }^{\circ} \mathrm{C}$ except for a few inactive fish at $20^{\circ} \mathrm{C}$. In contrast, most fish became motionless after longer incubation at $18{ }^{\circ} \mathrm{C}$. Fish were inactive at $16{ }^{\circ} \mathrm{C}$ and were unresponsive upon touching. Death appeared at $12{ }^{\circ} \mathrm{C}$ and below. The mortality increased with lower temperatures and longer incubation (data not shown). Thus, we used $18{ }^{\circ} \mathrm{C}$ to study cold shock responses to avoid mortality and undesired health problems.

To confirm that the exposure to $18{ }^{\circ} \mathrm{C}$ is able to elicit cold responses at the transcriptional level, we examined the change in gene expression of cold - inducible RNA binding protein (cirbp), a well-known cold inducible gene [11]. qPCR analysis showed that the cirbp expression was initially decreased at $2 \mathrm{~h}$ post induction (hpi), but significantly bounced back to about 2 folds at $4 \mathrm{hpi}$ and then declined to normal at $10 \mathrm{hpi}$ (Fig. 1a). It appeared that a 4-h incubation at $18{ }^{\circ} \mathrm{C}$ is sufficient to induce cold-dependent transcriptomic changes in zebrafish larvae.

qPCR analyses detected no notable change in expression of cirbp in samples before and immediately after transferring to $18{ }^{\circ} \mathrm{C}$ (data not shown). So the only time 0 control used was samples before cold shock. Larvae were then incubated at $28.5{ }^{\circ} \mathrm{C}$ (normal) or $18{ }^{\circ} \mathrm{C}$ (cold shock) and samples were taken from both conditions at 4 hpi (Fig. 1b). These experiments were repeated twice and a total of 6 different samples were subjected to small RNA and RNA NGS.

\section{Cold-induced miRNA sequencing profiles}

To clarify the roles of miRNA on transcriptomic plasticity in cold responses, we performed both small RNAseq and RNA-seq analyses in zebrafish larvae. Six RNA samples were extracted from three groups (control, normal and cold shock) as previously described, enriched for 18-30 bp small RNAs for the cDNA library construction and subjected to NGS using the SOLiD 5500 xl. A total of $39,560,685 ; 17,574,255$, and $27,708,966$ sequence reads from the first batch were obtained from the control, normal and cold shock sample, respectively (Fig. 2a, the total reads of the second batch were listed in Additional file 2: Figure S2A). The sequencing data was analyzed using the LifeScope ${ }^{\text {mu }}$ Genomic Analysis Software and normalized using the Partek Genomics Suite. The length distribution of each library reads were

Table 1 Cold impairs swimming and causes mortality in zebrafish larvae

\begin{tabular}{llllll}
\hline Incubation temperature & \multicolumn{1}{l}{ Hours Post Treatment } & & & \\
\hline${ }^{\circ} \mathrm{C}$ & 2 & 4 & 6 & Normal & 10 \\
28.5 & Normal & Normal & Normal & Normal & Normal \\
24 & Normal & Normal & Normal & Mostly normal but few & $\begin{array}{l}\text { Mostly normal but few } \\
\text { inactive larvae }\end{array}$ \\
20 & Normal & Normal & mostly normal but few & inactive larvae & 100 \\
18 (\% of inactive fish) & 83 & 95 & 96 & 100 & 12 \\
16 & All fish were inactive and showed unbalance moves when disturbed & 7.2 & 12 \\
12 (\% of motility) & 0 & 0 & 0 &
\end{tabular}

Four - day - old zebrafish larvae were incubated at decreasing temperatures from 28.5 to $12^{\circ} \mathrm{C}$. The mobility and viability were recorded every $2 \mathrm{~h}$ until $10 \mathrm{~h}$ post treatment 


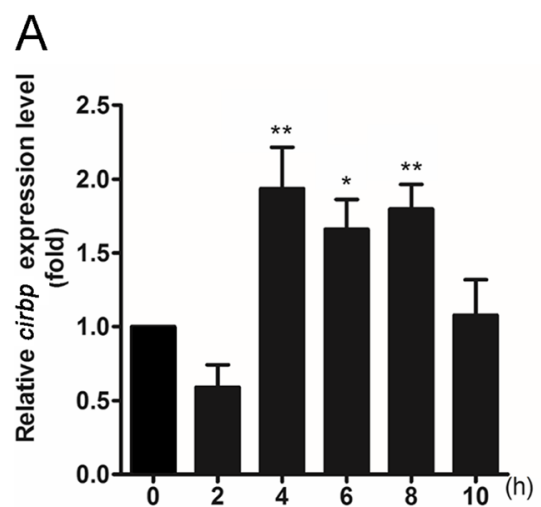

$\mathrm{B}$ Fig. 1 Cold shock treatment in zebrafish larvae. a Relative expression levels of cirbp gene in zebrafish larvae at $96 \mathrm{~h}$ post fertilization (hpf) were control at $0 \mathrm{~h}$ post transfer. $\mathbf{b}$ Scheme of cold exposure. Embryos were incubated at $28.5^{\circ} \mathrm{C}$ until $96 \mathrm{hpf}$ then transferred to

18 or $28.5^{\circ} \mathrm{C}$ for an additional $4 \mathrm{~h}$. Larvae before (control) and after treatments (normal and cold shock) were then collected for RNA-seq and miRNA-seq analyses as described in the Methods

ranged between 18 and $30 \mathrm{bp}$, and the major sizes peaked around 21 24 bp that matched the general size distribution of miRNAs (Additional file 2: Figure S1A, S2B). Abundant reads were located at chromosome 4, 10, 20, and 25 in different libraries (Additional file 2: Figure S1B, S2C). After filtering out short and adaptorcontaminated reads, about $17-19 \%$ miRNAs were identified. The reads and the percentage of different RNA components (miRNA, noncoding RNA, mRNA, novel RNA) were similar among samples (Fig. 2a). To examine the overall effects of cold shock on miRNAtome, the batches of data were normalized by reads count and subjected to principle component analysis to perform the quality check of samples as shown in Additional file 2: Figure S3. Then we mapped the sequencing reads to the miRBase v18, which contains 344 zebrafish miRNAs and found 261 known miRNAs. After normalizing two batches of data from three groups, the reads from different treatments were highly correlated (Fig. 2b). It suggests that the majority of miRNAs remained relatively constant during development and cold shock. Figure 2c shows the log plots of the fold of change in miRNA expression between treatments. We considered the fold of change $\geq 1.5$ folds to be significant. Transcriptomic profiles are changing rapidly during embryonic development. The altered miRNAs between control and normal were thought to be developmentdependent. After taking out development-dependent miRNAs, we identified 29 cold-induced miRNAs and 26 cold-repressed miRNAs (within the red boundary in Fig. 2d). The heat map of miRNAs with a change $\geq 1.5$ folds are shown in Fig. 2e and Additional file 3. The top 5 mRNAs affected are listed in Table 2 and the list of affected - miRNAs can be found in Additional file 4 .
Validation of miRNA-seq data with qPCR

We performed qPCR analysis to validate the changes of the cold-affected miRNAs (dre-mir-29b, the most up-regulated miRNA, and 9 other randomly selected miRNAs) and compared them to those observed in small RNA-seq results (Fig. 3a). The Pearson's correlation coefficient between two analyses is 0.87 . This is relatively high and suggests the reliability of small RNA-seq results (Fig. 3b).

\section{Function annotation for target genes of the cold- dependent miRNAs}

The cold-dependent target genes of each miRNA were predicted by TargetScanFish release 6.2 (http:// www.targetscan.org/fish_62/) with the total context+ score -0.3 as a cutoff [36]. We obtained 6263 and 5381 targets of cold-induced and -repressed miRNAs, respectively (Additional file 4). We then conducted the target genes functional annotation using The Database for Annotation, Visualization and Integrated Discovery (DAVID) Bioinformatics Resources 6.7 (http://david.abcc.ncifcrf.gov/) [37]. The top 10 biological processes were ranked according to $P$ value (Table 3 and Additional file 5). The $P$ value is an indication of gene enrichment in annotated biological process. The percentages of affected miRNA target genes with a value $\leq 0.005$ are illustrated in pie graphs (Fig. 3c). In general, both upand down-regulated miRNA target genes were enriched in processes like metabolism, transport, phosphorylation, development, signaling transduction, sexual determination and reproduction. In contrast, transcriptionrelated genes were only enriched in the up-regulated miRNA target genes, but cell movement and motilityrelated genes were abundant in the down-regulated miRNA target genes. 


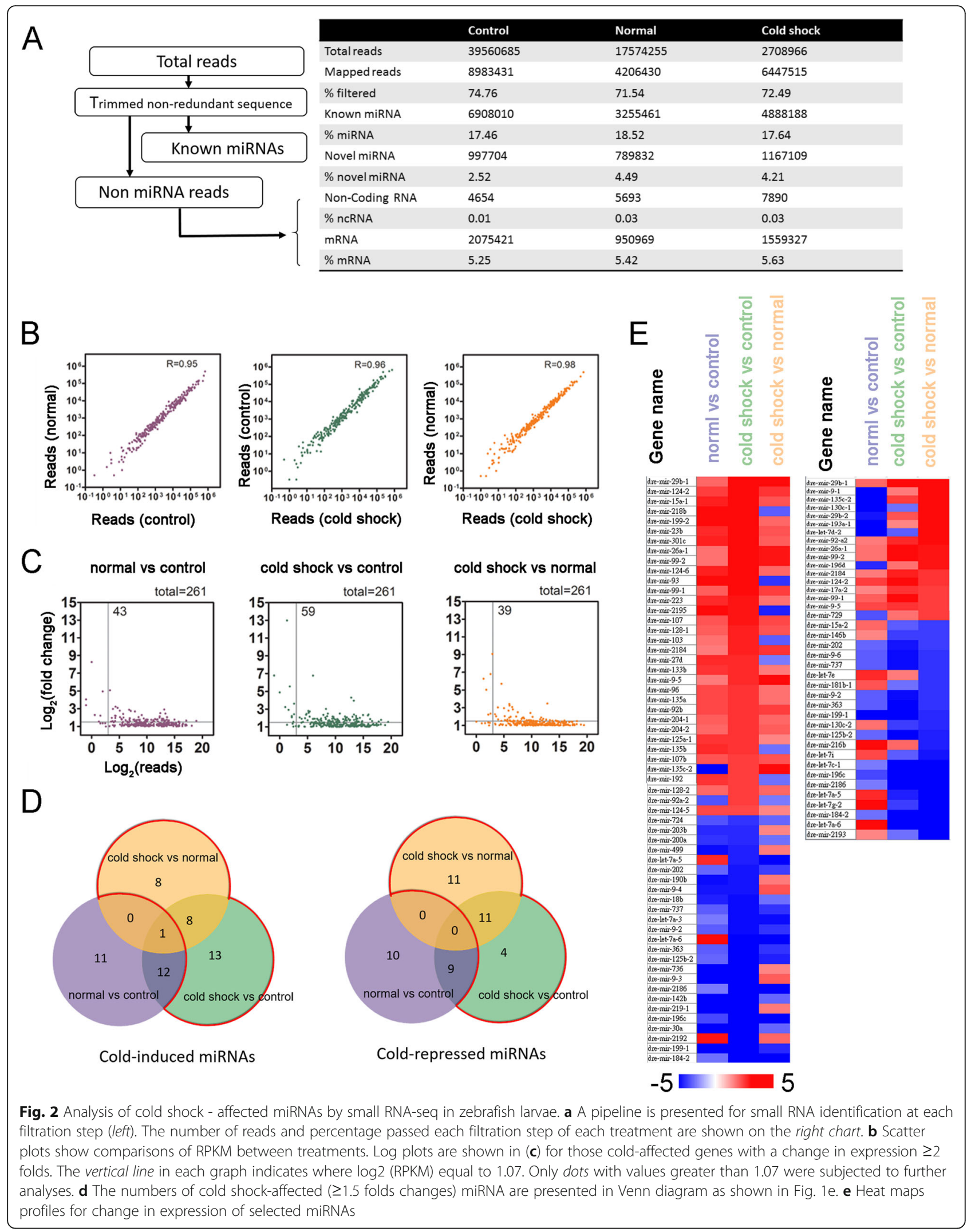


Table 2 Cold shock-induced differential expression of known miRNAs

\begin{tabular}{lll}
\hline miRNA & Mature miRNA sequence & Fold change \\
\hline Up-regulated miRNAs & \\
Dre-mir-29b-1 & UAGCACCAUUUGAAAUCAGUGU & 3.90 \\
Dre-mir-99-2 & AACCCGUAGAUCCGAUCUUGUG & 2.04 \\
Dre-mir-99-1 & AACCCGUAGAUCCGAUCUUGUG & 1.79 \\
Dre-mir-92a-2 & UAUUGCACUUGUCCCGGCCUGU & 1.75 \\
Dre-mir-2184 & AACAGUAAGAGUUUAUGUGCU & 1.73 \\
Down-regulated miRNAs & \\
Dre-mir-737 & AAUCAAAACCUAAAGAAAAUA & -1.71 \\
Dre-mir-9-2 & UCUUUGGUUAUCUAGCUGUAUGA & -1.75 \\
Dre-mir-363 & AAUUGCACGGUAUCCAUCUGUA & -1.79 \\
Dre-mir-125b-2 & UCCCUGAGACCCUAACUUGUGA & -1.91 \\
Dre-mir-199-1 & CCCAGUGUUCAGACUACCUGUUC & -2.79 \\
\hline
\end{tabular}

After eliminating miRNAs whose expression might be changed due to development process during incubation (control vs normal). The top 5 of 29 up- and 26 down-regulated known miRNAs (annotated in miRBase v18) are shown, along with their sequences and fold changes compared to the control groups

We also predicted the top 10 cold-induced and coldrepressed miRNA-mediated signaling pathways by using the Kyoto Encyclopedia of Genes and Genomes (KEGG) pathway database (Table 4 and Additional file 5). In the up-regulated group, most genes were involved in metabolism, including cysteine and methionine metabolism, fructose and mannose metabolism, glycerophospholipid metabolism, inositol phosphate metabolism and sphingolipid metabolism. One pathway was related to extracellular matrix-receptor interaction and the other one was related to biosynthesis of unsaturated fatty acids. In the cold-repressed group, many target genes were involved in biosynthesis and metabolism pathways, including chondroitin sulfate biosynthesis, synthesis and degradation of ketone bodies, O-Glycan biosynthesis, glycosphingolipid biosynthesis, biosynthesis of unsaturated fatty acids, sphingolipid metabolism and alanine, aspartate and glutamate metabolism. Some cold-repressed miRNA target genes were also found in the phosphatidylinositol, notch and lysozyme signaling pathways.

\section{Cold-induced RNA sequencing profile}

To validate our predicted miRNA targets, six RNA samples were extracted from zebrafish larvae, treated and processed as described previously to perform RNA-seq analysis using the SOLiD $5500 \mathrm{xl}$. The transcriptomic differences between three groups of fish were similarly analyzed as described in the miRNAtome analysis. Total numbers of reads and mapped reads of duplicates from three different treatments are shown in Fig. 4a. After normalizing two batches of data from three groups, the RPKM from different treatments (Fig. 4b) were highly correlated. It suggests that the majority of mRNAs remained relatively constant during development and cold shock. Figure 4c shows the log plots of the fold of change in RNA expression between treatments. We set the fold change $\geq 2$ folds and RPKM $\geq 0.1$ to be significant. After taking out development-dependent RNAs, we obtained 908 cold-induced genes and 463 cold-repressed genes (within the red boundary in Fig. 4d). The list of affected genes can be found in Additional file 6. The heat map of genes with a change $\geq 2$ folds are shown in Fig. 4e and Additional file 3. Among them, the genes related to circadian rhythm are indicated.

\section{Validation of the cold-induced changes in circadian clock genes with qPCR}

The circadian clock genes were also shown to be affected during cold acclimation in zebrafish [38]. However, their roles in the cold-induced response have not been elucidated. We first performed qPCR analysis to validate the changes of those circadian clock genes, which include per2, per1a, clock3, clock, arntl1a, arntl1b, nrld, cryla, and bhlhe41, under cold shock or normal conditions. The qPCR data was compared to those results by the RNA-seq (Fig. 4a). The Pearson's correlation coefficient between two analyses is 0.807 (Fig. 4b) that is relatively high and suggests the reliability of RNA-seq results.

\section{Function annotation of cold-dependent genes in transcriptome}

To reveal the biological interpretation of cold-dependent genes, we conducted the genes' functional annotation using DAVID Bioinformatics Resources 6.7 (http://david.abcc.ncifcrf.gov/) to find the enriched biological processes by the Gene Ontology (GO) term analysis. The $P$ value ranking indicates gene enrichment in annotated biological processes. The top 10 biological processes, such as regulation of RNA metabolic process, are listed in Table 5 and the number of genes belonging to each process are shown in Fig. 5c. The percentages of affected genes with a value $\leq 0.1$ are illustrated in a pie graph (Fig. 5d). In general, both up- and down-regulated genes were enriched in regulation of RNA metabolic processes, regulation of transcription, DNA-dependent and regulation of transcription. In contrast, protein amino acid phosphorylation, phosphate metabolic process, calcium ion transport, neuron differentiation and pattern specification process were only enriched in the upregulated genes. Response to DNA damage stimulus, cellular response to stress, cell fate commitment, embryonic morphogenesis, inorganic anion transport, anion transport and DNA repair were abundant in the down-regulated genes. 


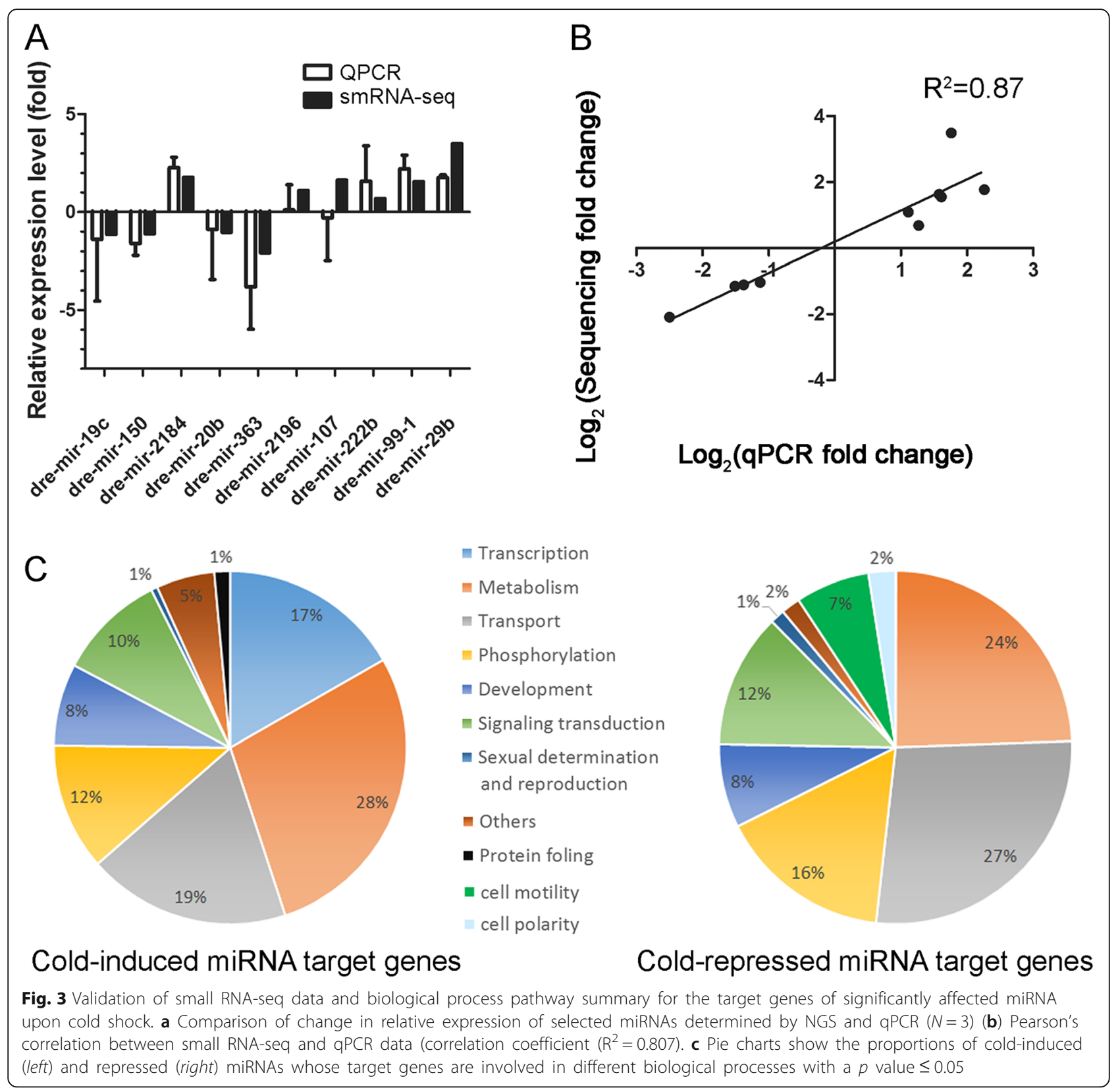

We also analyzed the cold-affected genes in the KEGG pathway database (Table 6) [39]. In the upregulated groups, most of the genes were involved in MAPK, hedgehog, GnRH and insulin signaling pathways. Other genes were enriched in immunityrelated pathways like retinoic acid-inducible gene 1 (RIG-I)-like receptor and oligomerization domain (NOD)-like receptor, circadian rhythm, melanogenesis and adhesion molecule. In the cold-repressed genes, only neuroactive ligand-receptor interaction and steroid biosynthesis were significantly enriched.

\section{Correlation of expression profiles of miRNA and mRNA}

To determine the correlation between cold-affected miRNAtome and transcriptome, we further analyzed the expression of cold-affected miRNAs and their target genes as shown in Additional file 7. The affected miRNAs should be negatively correlated with their target mRNAs. However, it is not always the case. Only $23.8 \%$ of up-regulated miRNAs resulted in down-regulation of target mRNAs. On the other hand, $75.4 \%$ of downregulated miRNAs caused up-regulation of target mRNAs. It appears that the release of miRNA inhibition 
Table 3 The top 10 biological processes for cold-related miRNA target genes

\begin{tabular}{lr}
\hline Biological Processes of & $P$ value \\
\hline Cold-induced miRNA target genes & \\
Regulation of transcription, DNA-dependent & $3.30 \mathrm{E}-04$ \\
Polyol metabolic process & $3.40 \mathrm{E}-04$ \\
Regulation of RNA metabolic process & $5.20 \mathrm{E}-04$ \\
Regulation of transcription & $6.70 \mathrm{E}-04$ \\
Regulation of small GTPase mediated signal transduction & $1.10 \mathrm{E}-03$ \\
lon transport & $1.10 \mathrm{E}-03$ \\
Glycerol metabolic process & $1.30 \mathrm{E}-03$ \\
Alditol metabolic process & $1.30 \mathrm{E}-03$ \\
Organophosphate metabolic process & $1.50 \mathrm{E}-03$ \\
Metal ion transport & $1.60 \mathrm{E}-03$ \\
Cold-repressed miRNA target genes & \\
Metal ion transport & $1.60 \mathrm{E}-04$ \\
Protein amino acid phosphorylation & $3.60 \mathrm{E}-04$ \\
Intracellular signaling cascade & $7.60 \mathrm{E}-04$ \\
lon transport & $8.50 \mathrm{E}-04$ \\
Cation transport & $1.80 \mathrm{E}-03$ \\
Potassium ion transport & $1.80 \mathrm{E}-03$ \\
Regulation of small GTPase mediated signal transduction & $1.80 \mathrm{E}-03$ \\
Organophosphate metabolic process & $3.10 \mathrm{E}-03$ \\
Monovalent inorganic cation transport & $3.60 \mathrm{E}-03$ \\
Regulation of Ras protein signal transduction & $4.70 \mathrm{E}-03$ \\
in annotation biological process & \\
barget genes of 29 up- and 26 down-regulated known miRNAs were & \\
bioinformatics resources 6.7 . The top 10 biologicted \\
genes and P values are shown & \\
&
\end{tabular}

may have a bigger impact on the expression of target mRNAs.

\section{Overexpression of per 2 assists in cold recovery in zebrafish larvae}

Biological processes of the Gene Ontology (GO) term analysis shows that circadian rhythm is enriched at the first category - regulation of RNA metabolic process as shown in Additional file 8. As described previously, the roles of circadian clock genes in the cold shock responses are still illusive. In the molecular level, the core clock genes, CLOCK and NPAS2/BMAL1, induce expression of repressors, PER1-3/CRY1-2 to form an interacting feedback loop to sustain the daily oscillation [40]. BHLHE41 plays a relative minor role that functionally resembles the inhibitor of the core clock gene complex and oscillates similarly with the PER1-3/CRY1-2 complex [41]. It has been reported that the dynamics of circadian clock genes are highly correlated with cell metabolism $[42,43]$. We hypothesized that the circadian gene-dependent changes in cell metabolism may
Table 4 The top 10 KEGG pathways for cold-related miRNA target genes

\begin{tabular}{ll}
\hline KEGG pathway of & $P$ value* \\
\hline Cold-induced miRNA target genes & \\
Cysteine and methionine metabolism & 0.0027 \\
ECM-receptor interaction & 0.0035 \\
Fructose and mannose metabolism & 0.005 \\
Biosynthesis of unsaturated fatty acids & 0.011 \\
Glycerophospholipid metabolism & 0.011 \\
Inositol phosphate metabolism & 0.014 \\
Sphingolipid metabolism & 0.022 \\
Melanogenesis & 0.028 \\
GnRH signaling pathway & 0.029 \\
Circadian rhythm & 0.034 \\
Cold-repressed miRNA target genes & \\
Chondroitin sulfate biosynthesis & 0.00081 \\
Synthesis and degradation of ketone bodies & 0.0092 \\
Phosphatidylinositol signaling system & 0.017 \\
Sphingolipid metabolism & 0.019 \\
Notch signaling pathway & 0.023 \\
O-Glycan biosynthesis & 0.025 \\
Lysosome & 0.03 \\
Alanine, aspartate and glutamate metabolism & 0.035 \\
Biosynthesis of unsaturated fatty acids & 0.036 \\
& 0.038 \\
\hline
\end{tabular}

*calculated by Fisher Exact which is adopted to measure the gene-enrichment in annotation KEGG pathway

Target genes of 29 up- and 26 down-regulated known miRNAs were predicted by TargetScan (Additional file 5). The targets genes were annotated by DAVID bioinformatics resources 6.7. The top 10 KEGG pathway of the target genes and $P$ values are shown

help in cold resistance. To test this hypothesis, we selected three circadian clock genes, arntl1a (bmal), bhlhe41 and per2, which had different levels of change (1.14, 2.4 and 12.6 folds, respectively) upon cold shock, to perform functional assays. We designed overexpressing plasmids containing Tol2, 5XUSA element, different circadian clock gene coding region sequence and $\mathrm{H} 2 \mathrm{AmCherry}$ indicator. The circadian clock gene and mCherry were intervened with a $2 \mathrm{~A}$ peptide sequence for separation upon translation. Different plasmids were separately injected into 1-cell embryos with gal4 mRNAs (Fig. 6a). The ectopic expression of these plasmids were screened by the H2AmCherry fluorescence under epifluorescent microscope as an indirect indication of circadian clock gene overexpression (Fig. 6b). To validate this, we performed qPCR analyses and found that the expressions of bmal, per 2 and bhlhe 41 were increased to $18.86 \pm 9.1,12.85 \pm 11.17$ and $5.02 \pm 1.88$ folds, respectively (Fig. 6c, $N=3$ ). These fish with transient expression of circadian clock genes were then used for the cold tolerance assay. 


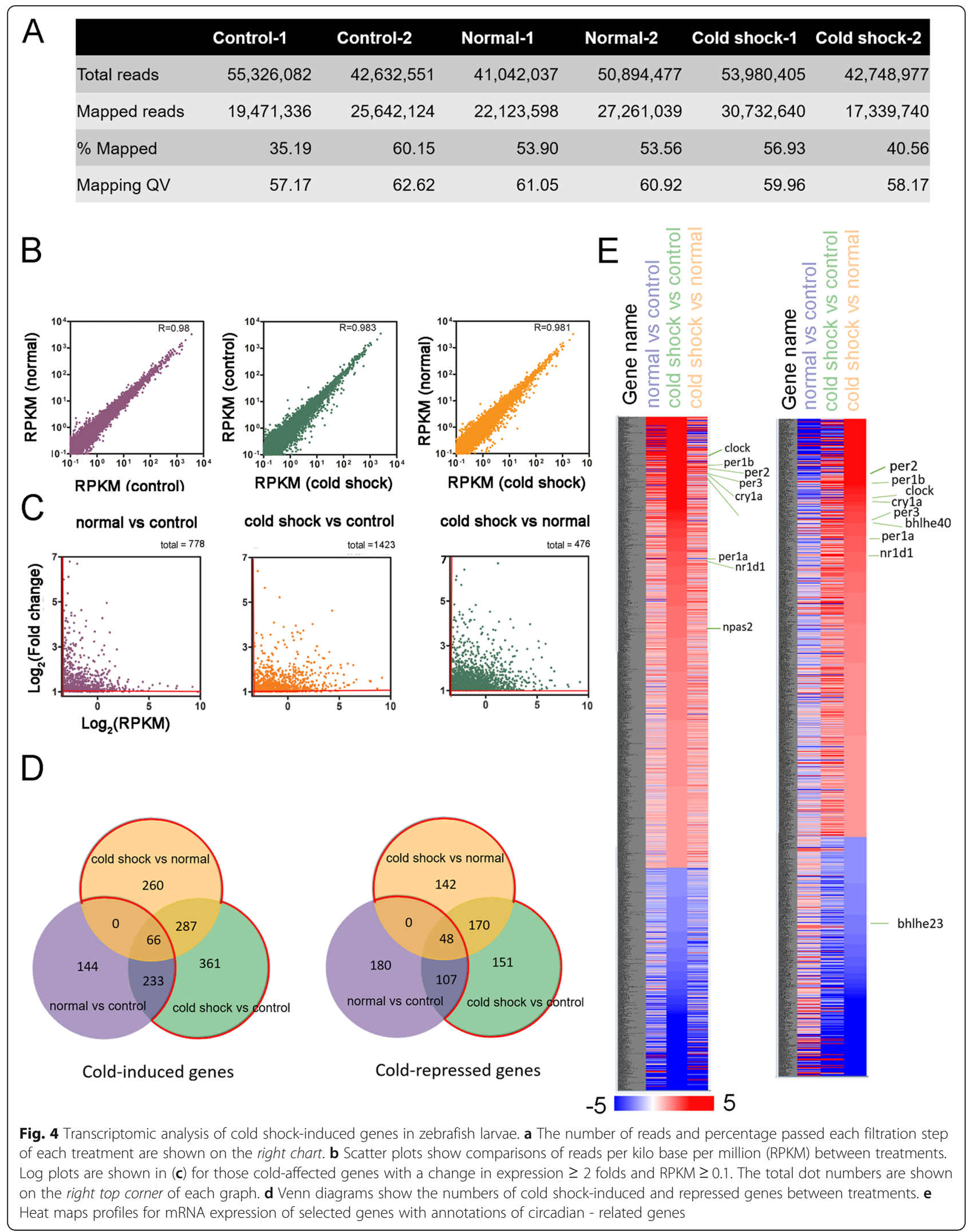


Table 5 The top 10 biological processes for cold-related genes

\begin{tabular}{ll}
\hline Biological Processes of & $P$ value $^{*}$ \\
\hline Cold-induced genes & \\
Regulation of RNA metabolic process & $1.50 \mathrm{E}-03$ \\
Regulation of transcription, DNA-dependent & $1.80 \mathrm{E}-03$ \\
Protein amino acid phosphorylation & $1.90 \mathrm{E}-03$ \\
Phosphate metabolic process & $2.10 \mathrm{E}-03$ \\
Rgulation of transcription & $5.40 \mathrm{E}-03$ \\
Phosphorylation & $6.80 \mathrm{E}-03$ \\
Transcription & $9.50 \mathrm{E}-03$ \\
Calcium ion transport & 0.01 \\
Neuron differentiation & 0.015 \\
Pattern specification process & 0.019 \\
Cold-repressed genes & \\
Response to DNA damage stimulus & 0.01 \\
Cellular response to stress & 0.018 \\
Regulation of transcription & 0.019 \\
Cell fate commitment & 0.027 \\
Regulation of RNA metabolic process & 0.03 \\
Embryonic morphogenesis & 0.033 \\
Inorganic anion transport & 0.037 \\
Regulation of transcription, DNA-dependent & 0.04 \\
Dnion transport & 0.04 \\
DNA repair & 0.047
\end{tabular}

*calculated by Fisher Exact which is adopted to measure the gene-enrichment in annotation biological process

The cold-related genes (Additional file 6) were annotated by DAVID bioinformatics resources 6.7. The top 10 biological processes of the cold-related genes and $P$ values are shown

Zebrafish swimming behavior has been used as an indicator for cold tolerance [44]. We first cultured the circadian clock gene overexpressing fish under a $14 \mathrm{~h} / 10 \mathrm{~h}$ dark light cycle for 5 days and incubated in $18{ }^{\circ} \mathrm{C}$ for $4 \mathrm{~h}$. We then transferred fish to a $28.5{ }^{\circ} \mathrm{C}$ observation chamber to record the spontaneous swimming behavior from 0 to 30 mins after transfer (Fig. 6d). The moving paths at different intervals after cold recovery $(0-10$, 10-20 and 20-30 min) were recorded and analyzed according to the intensities of four classes of swimming activity (Fig. 6e). The per 2 and bhlhe 41 overexpressing fish had higher swimming activity than those of control and arntl1a overexpressing fish at 10-20 mins interval (\% of moving fish: Ctrl: $23 \pm 7 \%, n=40$; Bmal: $35 \pm 7 \%, n=34$; Per2: $39 \pm 15 \%, n=35$, Bhlhe41: $42 \pm 15 \%, n=36$ ). The per2-overexpressing fish exhibited the most active swimming behavior during the 20-30 min interval (\% of moving fish: Ctrl: $44 \pm 9.2 \%, n=32$; Bmal: $42 \pm 12 \%$, $n=36$; Per2: $76 \pm 12 \%, n=30$; Bhlhe41: $53 \pm 10 \%, n=34$ ) as shown in Fig. $6 f$.
To examine cell metabolism, we measured the change in glucose concentration in the circadian clock gene overexpressing larvae. In control and bmal (arntlla)overexpressing fish, the glucose concentration was gradually increased during cold shock. In contrast, the bhlhe41- and per2-overexpressing fish had a higher basal glucose level and the level was not significantly changed during cold shock (Fig. 6g).

\section{Dre-mir-29b targets per2 mRNA and regulates its expression dynamically during cold treatment}

The increase in cold tolerance in per2-overexpressing fish suggests a pivotal role of circadian clock genes against cold stress. Comparing the expression level of miRNA-seq and mRNA-seq of gene of interest, we identified several cold-dependent miRNAs that may regulate circadian clock genes (Tables 7 and 8 ). We selected dremiR-29b to illuminate how miRNA affect transcriptome plasticity during cold shock (Fig. 7a). Dre-miR-29b is the top one cold-induced miRNA with a predicted target of per2. To address this, we injected pEGFPC1-per2 3'UTR with or without dre-mir-29b morpholino oligonucleotides (MO) (Fig. 7b). The co-injection of mir-29b MO significantly enhanced the \% of embryos showing the fluorescence from the pEGFPC1-per2 3'UTR (51.9\%, $n$ $=95, N=3$ ) compared to that of plasmid only embryos $(8.65 \%, n=49, N=3)$ as shown in Fig. 7c. This data suggests that dre-mir-29b MO can prohibit dre-mir-29b targeting per 2 mRNAs.

To further study the dynamic expression of per 2 and dre-mir-29b during cold shock, we measured their expression levels at a shorter duration (every 2-4 h) until 24 hpi (Fig. 7d). It appeared that both dre-mir-29b and per 2 expression was elevated at 4 hpi. The per 2 expression declined afterward. In contrast, the dre-mir-29b expression increased afterwards, but a sudden drop was observed at 24 hpi. These results suggested that dre-mir$29 b$ may act to control the per 2 expression-induced by cold shock.

\section{Discussion}

Cold shock is a general, but harmful, environmental stress for ectothermic animals. We performed high throughput small RNA-seq and RNA-seq experiments to investigate potential regulatory mechanisms for coldinducible responses and to characterize cold-affected miRNAs and genes in zebrafish larvae. We found 29 cold-induced miRNAs and 26 cold-repressed miRNAs in the microRNAtome analysis. We also discovered 908 cold-induced genes and 468 cold-repressed genes in the transcriptome analysis. Among them, circadian clock genes and others were enriched in both analyses. Thus, we overexpressed one of the cold-induced core clock gene per 2 and showed a notable increase in cold 


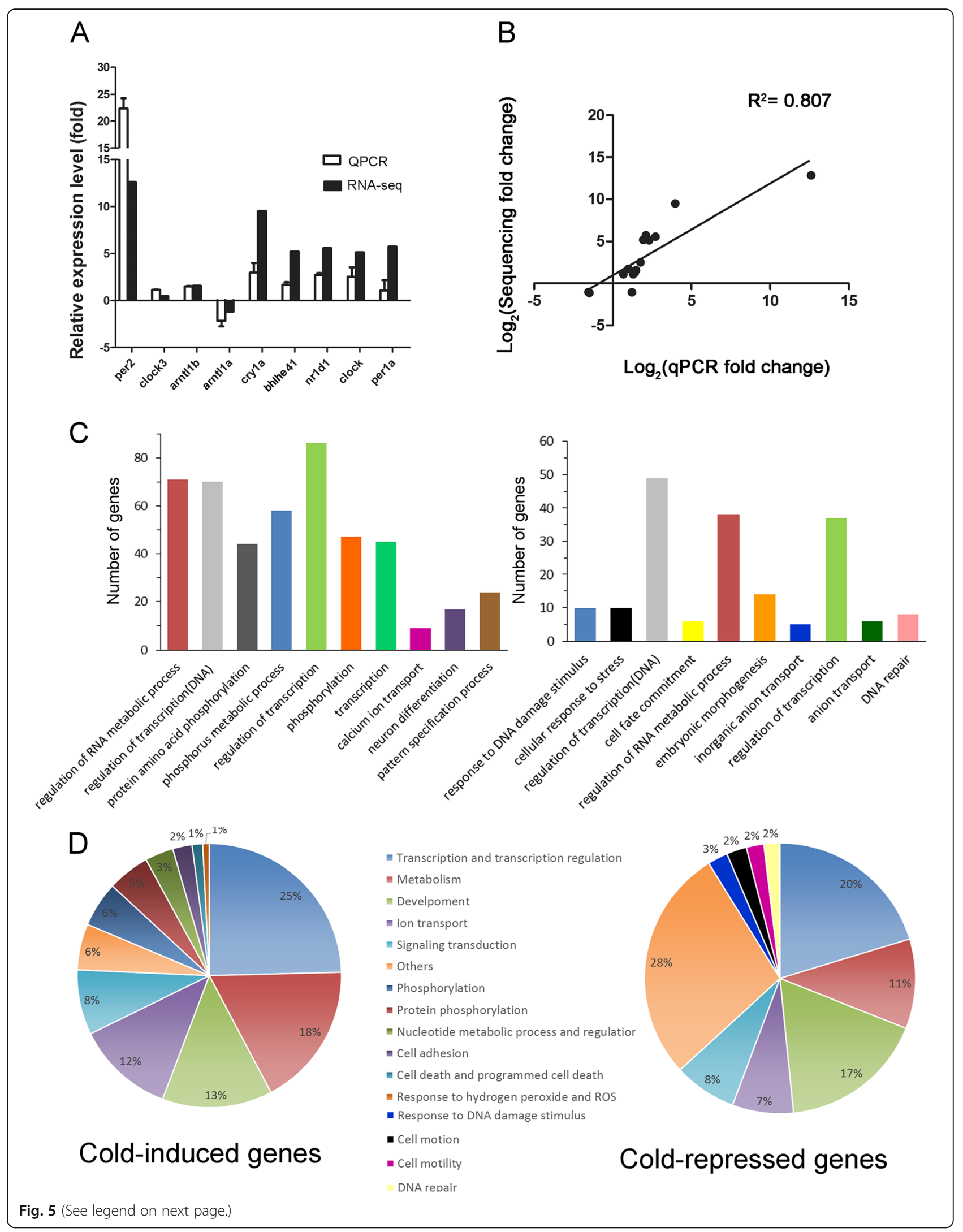


(See figure on previous page.)

Fig. 5 Validation of RNA-seq data and biological process pathways summary of the genes significantly changed upon cold shock. a Comparison of change in relative expression of circadian clock genes determined by NGS and QPCR $(N=3)$. b Pearson's correlation result between RNA-seq and qPCR data. (Correlation coefficient $=0.807$ ) (c) Numbers of cold-induced (left) and repressed (right) genes belong to the top 10 biological processes. $\mathbf{d}$ Pie charts show the proportions of cold-induced (left) and repressed (right) genes which involve in different biological processes with a $p$ value $\leq 0.05$

tolerance in zebrafish larvae. We further validated the targeting of per 2 by its associated miRNA, dre-mir-29b, which was also induced by cold shock. This suggests that miRNA can serve as a fine tuner for regulating the transcription of cold-inducible genes during cold stress.

An acute temperature drop may result in lethality in aquatic animals. Therefore, we first determined a permissible temperature $\left(18{ }^{\circ} \mathrm{C}\right)$ that could induce adaptive cold responses without deleterious effects on fish. Cirbp, a cold shock-inducible protein, is served as a molecular indicator for cold shock. Cirbp acts as a RNA chaperone to unfold RNA secondary structures to facilitate translation. The elevation of cirbp expression has been known to be a universal marker upon cold exposure [1]. We measured the cirbp expression by qPCR and found that $4 \mathrm{~h}$ is the minimum duration which could cause significant gene induction. Furthermore, transcriptome and miRNAtome profiles change rapidly during the larvae stage, however, it was generally ignored in previous studies $[15,38]$. Therefore, we ruled out the genes, which might be changed during development to be sure that the up- and down- regulated genes selected are truly cold shock induced.

Table 6 The top 10 KEGG pathways for cold-related genes

\begin{tabular}{|c|c|}
\hline KEGG pathway of & $P$ value* \\
\hline \multicolumn{2}{|l|}{ Cold-induced genes } \\
\hline MAPK signaling pathway & 2.60E-05 \\
\hline Circadian rhythm & 2.60E-04 \\
\hline RIG-I-like receptor signaling pathway & 0.011 \\
\hline GnRH signaling pathway & 0.025 \\
\hline Cell adhesion molecules (CAMs) & 0.026 \\
\hline Adherens junction & 0.034 \\
\hline Melanogenesis & 0.036 \\
\hline Hedgehog signaling pathway & 0.049 \\
\hline NOD-like receptor signaling pathway & 0.056 \\
\hline Insulin signaling pathway & 0.057 \\
\hline \multicolumn{2}{|l|}{ Cold-repressed genes } \\
\hline Neuroactive ligand-receptor interaction & $3.90 \mathrm{E}-03$ \\
\hline Steroid biosynthesis & $6.50 \mathrm{E}-03$ \\
\hline
\end{tabular}

The cold-affected genes and miRNAs are involved in many crucial biological processes. The most dominate pathways are attributed to transcription regulation, protein phosphorylation and transport. According to previous studies, both cold acclimation and cold shock could affect RNA transcription [11, 15, 45]. In our RNA-seq data, around $20 \%$ cold-regulated genes encode transcription factors such as her, hsf2, hsf1, dmrt3a, poulf1, atf3, gata1a, cebpb, fosl1, bhlhe41, bhlhe40, nr1d4b, nr1d4a, nrld1 and nrld2a. Among these genes, bhlhe41, bhlhe40, nr1d4b, nr1d4a, nr1d1, nr1d2a have been reported to be enriched in cold-induced carp larvae [11], suggesting transcription of certain genes should be induced by the cold in the fish larvae stage. The protein phosphorylation landscape is changed during cold treatment in aquatic creatures [46]. In the same token, we also observed the up-regulation of many kinase proteins such as MAP kinase interacting serine/threonine kinase 1 cascades, calcium/calmodulin-dependent protein kinase, protein kinase $\mathrm{C}$ and casein kinase 1 . Furthermore, we found the changes in expression of multiple ion transporters (atp $2 a 2 a, a t p 2 a 2 b$, atp $2 b 3 a, c a c n b 2 a, c a c-$ $n a 1 c$, ryr $1 b$, and slc8a2b). It implies that cold shock may inhibit ion pumps and channels that results in imbalance of osmoregulation in fish $[47,48]$. Studies also revealed that cold can affect the oxidation reduction processes $[15,45]$. However, these processes were not significantly changed in our analyses, which may reflect different cold treatment regime or animals used. In general, our results are in good agreement with previous reports.

Circadian clock genes, including bhlhe40, npas2, $n r 1 d 1, n r 1 d 4$, per $1 a$, per $1 b$ and per 2 , are under the regulation of RNA metabolic process. The RNA metabolic process is the No. 3 and No. 1 in the cold-induced miRNA target genes (Table 3) and the cold-induced genes (Table 5), respectively. We analyzed genes belonging to the regulation of RNA metabolic process by using the KEGG pathway analysis and found that circadian rhythm is one of two most enriched pathways as shown in the second worksheet of Additional file 8. It gains further proof that circadian rhythm is listed as the No. 2 pathway for all cold-induced genes analyzed as shown in Table 6. Collectively, these results indicate circadian clock genes are notably affected by cold shock.

A group of circadian clock genes, and their associated miRNAs were upregulated upon cold shock (Tables 7 and 8). Similar upregulation of circadian clock genes by 
A

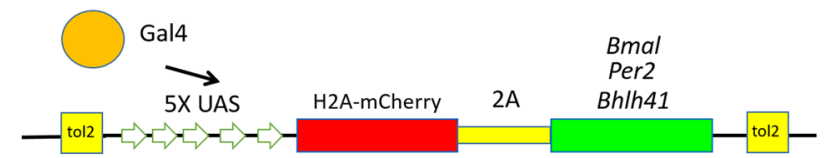

B

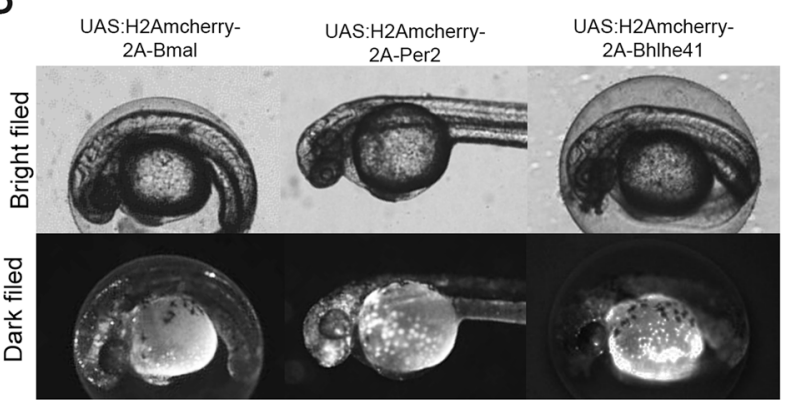

C

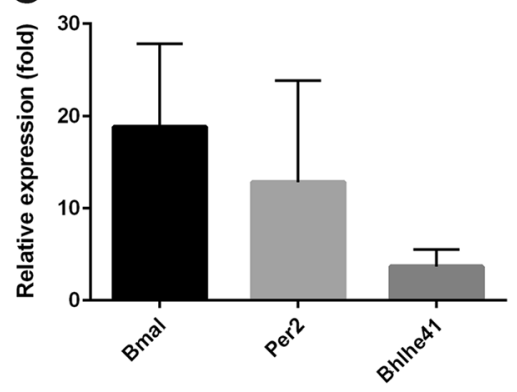

D

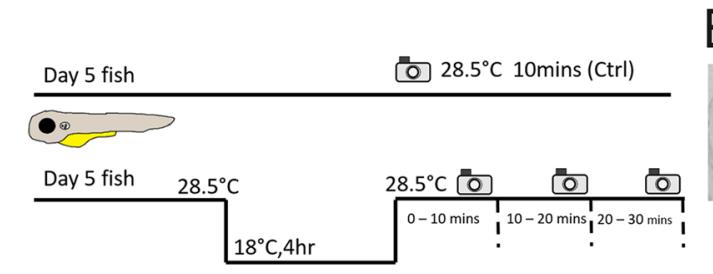

E

F

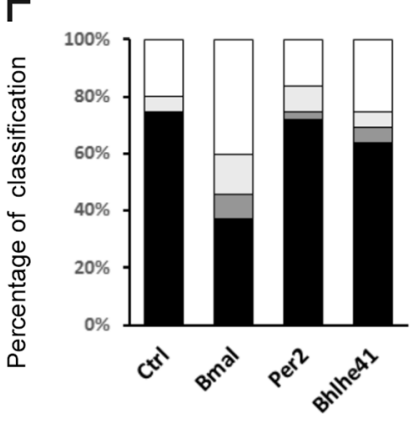

G

$$
\text { Ctrl }
$$

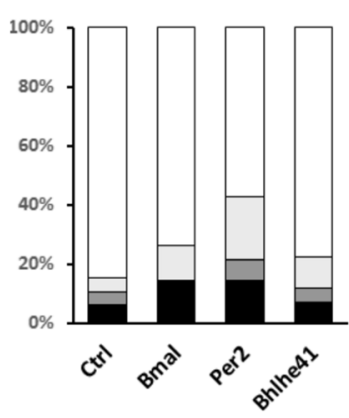

0-10 mins
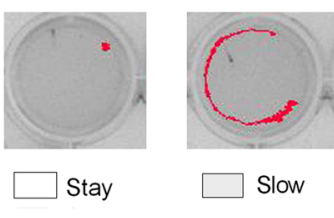

$\square$ Slow
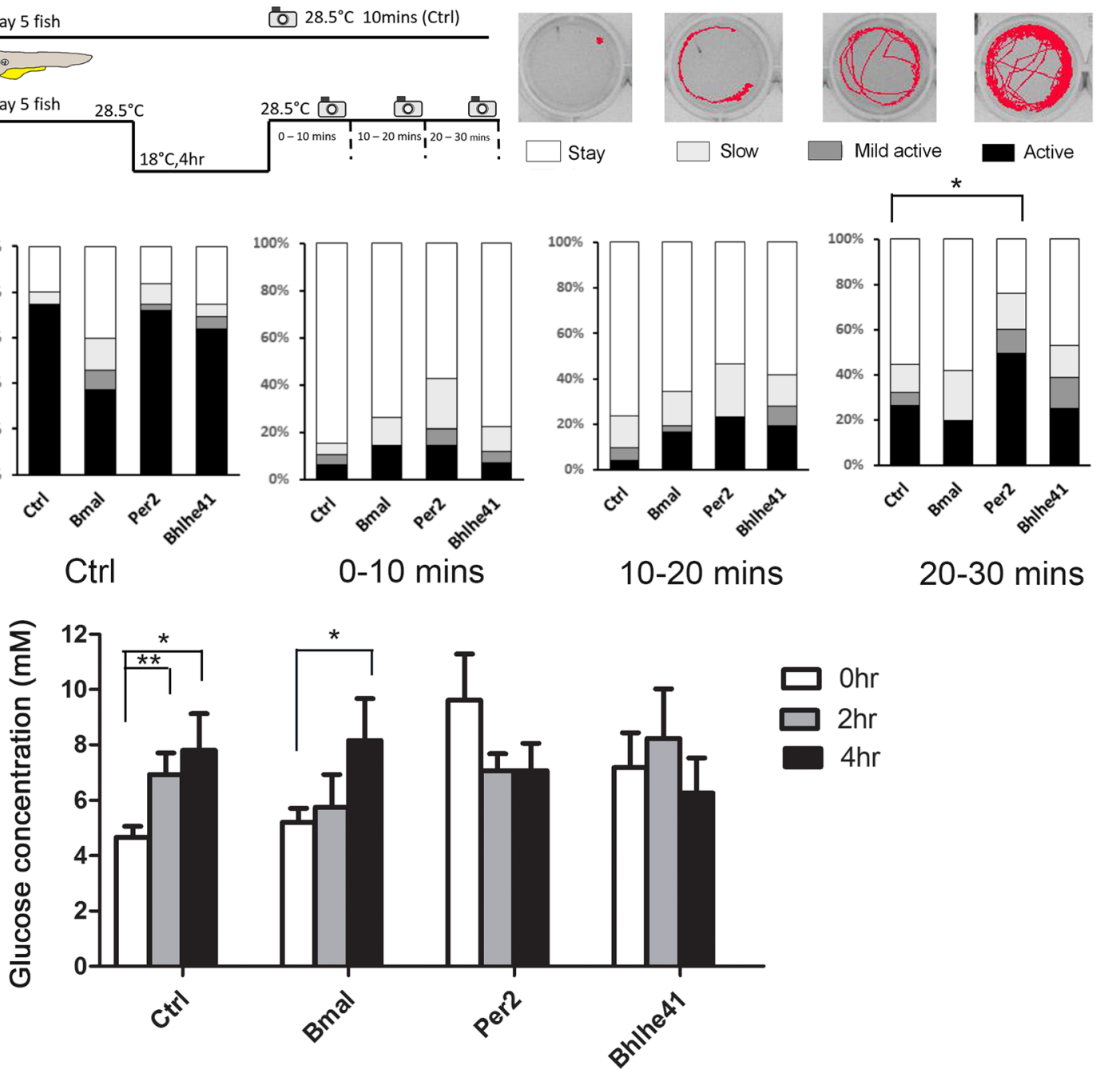

20-30 mins

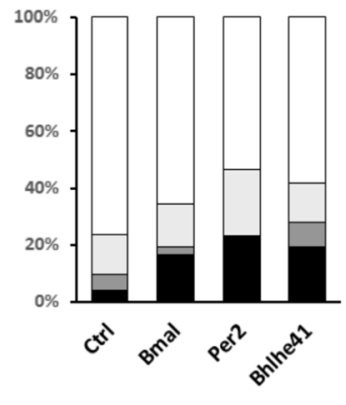

Fig. 6 (See legend on next page.) 
(See figure on previous page.)

Fig. 6 Overexpression of core clock gene per2 or bh/he41 increases cold tolerance. a The design of the Gal-UAS driven circadian clock gene expression construct is shown. H2AmCherry (red) is placed upstream of clock gene (green) that will be cleaved by a 2A peptide (yellow). b 1-Cell embryos were injected with indicated plasmids and examined under bright (upper row) and dark filed (bottom row through a rhodamine filter cube). Mosaic expression of mcherry indicates the expression of bmal, per2, or bhlhe41 in 48 hpf embryos. c Normalized expression level of clock genes compared to control embryos at 5 days post fertilization by qPCR $(N=3)$. d Scheme of cold recovery assay at 5 days post fertilization fish. e Classifications of swimming track recorded for 10 mins. $\mathbf{f}$ Quantitative analysis of swimming patterns for zebrafish larva transient expressed without (control) and with designated circadian clock gene at different time periods after recording. $\mathbf{g}$ Glucose concentration were measured in $4 \mathrm{dpf}$ zebrafish larval lysate collected at different time point after cold shock. ${ }^{*} P \leq 0.05,{ }^{* *} p \leq 0.01$

cold stress have also been observed in previous studies $[15,38]$. It suggests a possible involvement of circadian associated regulation during cold acclimation and cold shock. However, direct evidence for a role of circadian clock genes in cold responses is still lacking. Circadian rhythm controls the daily rhythm for an organism in behavior, physiology and biochemistry [40]. The molecular basis of this oscillation consists of interaction between positive and negative feedback loops. At the molecular level, the circadian rhythm is governed by a set of transcription activators, CLOCK and NPAS2/BMAL1, which induce expression of repressors, PER1-3/CRY1-2, to form an interacting feedback loop [49]. BHLHE41 functions to inhibit CLOCK:BMAL1 transactivation of the clock gene Per1 to resembles the negative feedback loop of PER/CRY complex [41]. In addition to light, circadian rhythm could also be entrained by temperature and feeding behavior [50]. Zebrafish core clock genes, including cry $2 a$, cry 3 and per4, can be entrained by lower temperature but not per2 [51]. This implies the elevation of per2 in our study may play a novel biological function in the cold stress response. Here, we successfully demonstrate that overexpression of core clock gene per 2 can increase cold tolerance in zebrafish larvae upon cold shock, but not bmal. Previous studies showed that

Table 7 Cold-affected circadian clock genes and associated miRNAs

\begin{tabular}{|c|c|}
\hline Circadian rhythm & miRNA \\
\hline $\begin{array}{l}\text { Basic helix-loop-helix domain containing, } \\
\text { class B, } 3 \text { like }\end{array}$ & $\begin{array}{l}\text { Dre-mir-92, dre-mir-107, } \\
\text { dre-mir-103 }\end{array}$ \\
\hline Cryptochrome 3 (cry3) & Dre-mir-29 \\
\hline $\begin{array}{l}\text { Aryl hydrocarbon receptor nuclear } \\
\text { translocator-like } 1 \mathrm{~b} \text { (arnt11b) }\end{array}$ & Dre-mir-99 \\
\hline Clock homolog 3 (clock3) & $\begin{array}{l}\text { Dre-mir-29, dre-mir-96, } \\
\text { dre-mir-499 }\end{array}$ \\
\hline Period homolog 2 (per2) & Dre-mir-29,dre-mir-18 \\
\hline Period homolog 1a (per1a) & Dre-mir-135 \\
\hline Nuclear receptor subfamily 1 (nrld1) & Dre-mir-204 \\
\hline $\begin{array}{l}\text { Basic helix-loop-helix family, } \\
\text { member e40 (bh/he40) }\end{array}$ & Dre-mir-128, dre-mir-181 \\
\hline Casein kinase 1, epsilon & Dre-mir-130, dre-mir-301 \\
\hline Casein kinase 1 , delta a & Dre-mir-29 \\
\hline
\end{tabular}

knockout per2 impairs gluconeogenesis [52] but bmal null mice increased gluconeogenesis [53], suggesting two proteins may play opposite role in glucose metabolism. The per2 gene may have a glucocorticoid response element that responds to glucocorticoid hormones to increase blood glucose [54]. The elevation in plasma glucose is enhanced by the glucocorticoid/cortisol-mediated gluconeogenesis, which is a common stress response in aquatic creatures in response to cold [55]. We observed the basal glucose level remains high in transgenic zebrafish larvae overexpressing per 2 , which suggests that the elevation of per 2 expression level may counter cold stress by maintaining a higher gluconeogenesis. Per2 is also known to control lipid metabolism by direct regulation of PPAR [56]. Whether Per2 also exerts its cold-defending function via enhanced lipid metabolism still awaits further investigation. Furthermore, bhlhe41-overexpressing fish also showed better

Table 8 Cold-affected miRNAs and corresponding circadian clock genes

\begin{tabular}{lll}
\hline MicroRNA & Up/Down & Target \\
\hline Dre-mir-29b & Up & Per2, Per3, Cry3, Ck1,Clock3 \\
Dre-mir-135 & Up & Per1a, Nr1d4a \\
Dre-mir-99 & Up & Arntl1b \\
Dre-mir-92a & Up & Bhlhe41 \\
Dre-mir-96 & Up & Clock3 \\
Dre-mir-204 & Up & Nr1d1 \\
Dre-mir-128 & Up & Bhlhe40 \\
Dre-mir-107 & Up & Bhlhe41 \\
Dre-mir-2192 & Down & Clock \\
Dre-mir-18 & Down & Per2, Per3,Bhlhe41 \\
Dre-mir-202 & Down & Nr1d2b \\
Dre-mir-181 & Down & Bhlhe40, Nr1d12a \\
Dre-mir-363 & Down & Nr1d2a, Bhlhe41 \\
Dre-mir-130 & Down & Ck1E, Nr1d12a \\
Dre-mir-301 & Down & Ck1E \\
Dre-mir-184 & Down & Crylb \\
Dre-mir-499 & Down & Clock3, Nr1d14b \\
\hline
\end{tabular}

Up/Down: Significantly up-regulated or down-regulated miRNAs are listed, the comprehensive Targeting clock genes are listed at the right column 

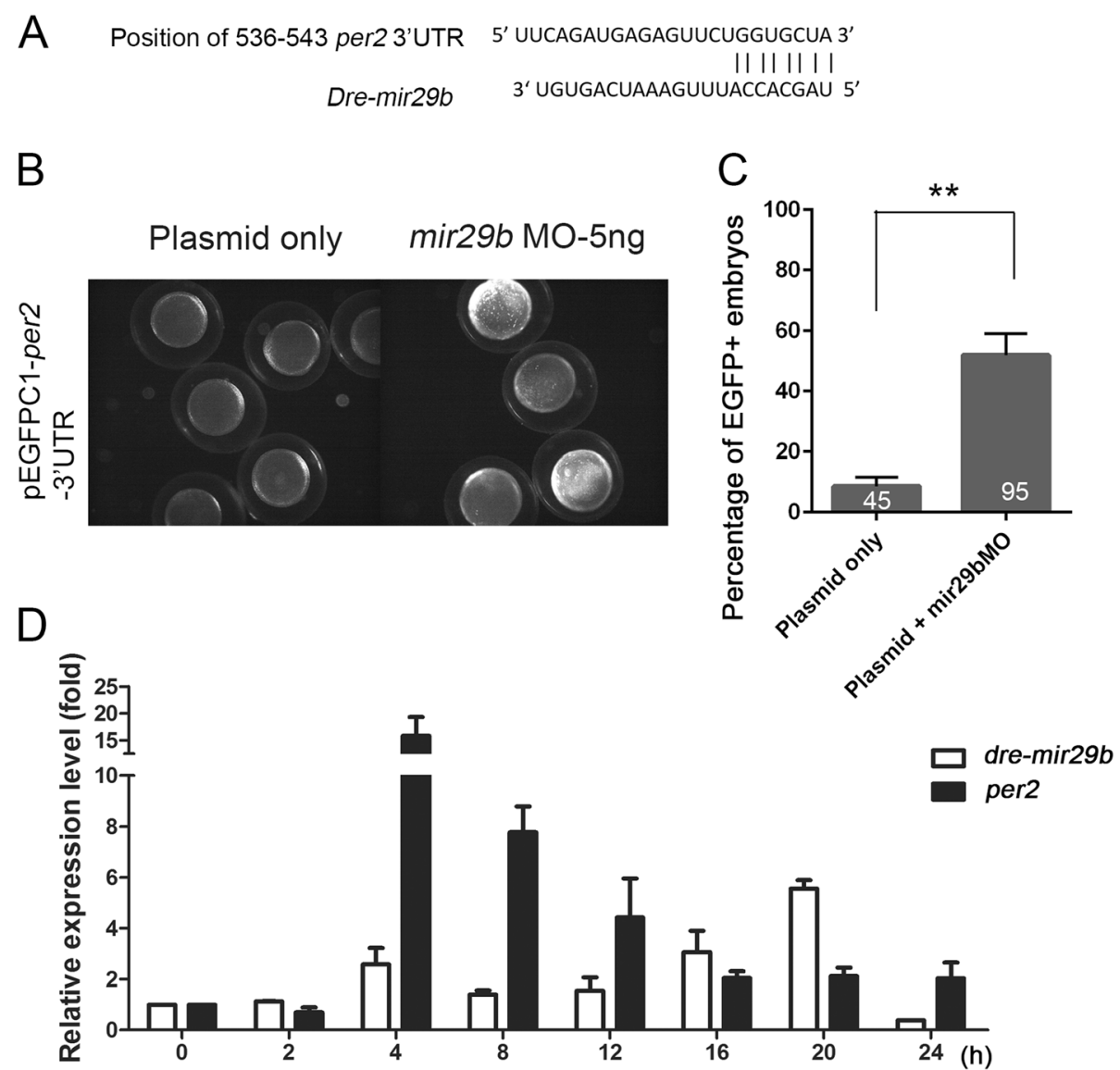
region ( $3^{\prime} U T R$ ) containing the complementary nucleotides of dre-mir-29b is shown. b 1-cell embryos were injected 50 pg pEGFPC1 bearing $3^{\prime} U T R$ of per2 target site in the presence or absence of $5 \mathrm{ng}$ mir-29b MO and examined at $90 \%$ epiboly stage under epifluorescent microscopy using a FITC cube. c The percentages of EGFP - positive embryos injected without or with dre-mir-29b are shown. The numbers of embryos used in each treatment are presented at the bottom of each column. $(N=3),{ }^{* *} p \leq 0.01$. $\mathbf{d}$ Embryos were treated with cold shock, lysed at designated time post cold shock, and their RNAs were extracted for qPCR analysis for dre-mir-29b (open bar) and per2 (filled bar)

cold tolerance in locomotor activity and retain higher glucose level. It will be interesting to study whether the inhibition of CLOCK/BMAL complex would enhance cold tolerance.

miRNAs play a key role in cellular responses to the changing environment. Upon stress, cells also tend to restore or reprogram their transcriptomes to counter the changes modulated by miRNAs [27]. It implies a plausible role of miRNAs in mediating cold responses. However, Yang et al. previously reported that cold acclimation trigger changes in some miRNA profiles in adult zebrafish brains and those miRNAs have poor associations to the affected genes. They concluded that microRNAs play a minor role in mediating transcriptome plasticity during cold stress [30]. It appears to be contradictory to our finding that the affected miRNAs have predicted target genes involved in cold-inducible biological processes, including melanogenesis, GnRH signaling pathway and circadian rhythm.
To clarify the interaction between per 2 and associated dre-mir-29b, we examined their dynamic expression during cold shock and found good correlations for dremir-29b to serve as an inhibitor of per 2 expression. The dynamic pattern between miRNAs and target genes nicely matches the "pulse model" as described by Leung et al. [27]. As miRNAs accumulate over time, miRNAs exert their suppressive effect on the mRNA targets, causing a decrease in mRNA target expression. The poor correlation between cold-affected miRNAs and mRNAs observed previously [30] might be due to the time of sample collection or the use of different tissues (brain tissue vs. whole larvae used here). The duration of stress also determines if cells can re-establish homeostasis or initiate the mechanism to acclimate to the new environment [57]. The discrepancy between two studies may also be resulted from different machineries used in two different stress levels (cold acclimation v.s. cold shock). 


\section{Conclusions}

Here, we demonstrate for the first time that miRNAs can be fine turners for regulating genomic plasticity against cold shock. We further showed that the fine tuning of core clock gene per2 via its associated miRNA, dre-mir-29b, can enhance the cold tolerance of zebrafish larvae. The circadian clock genes may thus serve as targets for designing novel strategies to face cold challenges in aquaculture.

\section{Additional files}

Additional file 1: Lists of primers. (XLSX $10 \mathrm{~kb}$ )

Additional file 2: Reads length, chromosome distribution and BCA analysis of smRNA-seq. (DOCX $2284 \mathrm{~kb}$ )

Additional file 3: Heat maps of cold-related miRNAs and genes. (XLSX $105 \mathrm{~kb}$ )

Additional file 4: Lists of cold-related miRNAs and target genes. (XLSX $530 \mathrm{~kb})$

Additional file 5: $\mathrm{GO}$ term analysis of miRNA corresponding target genes. (XLSX $135 \mathrm{~kb}$ )

Additional file 6: List of cold-related genes and GO term analysis. (XLSX $30 \mathrm{~kb})$

Additional file 7: Expression profiles of miRNAs and their target mRNAs. (XLSX $104 \mathrm{~kb}$ )

Additional file 8: KEGG pathway analysis of dre-mir-29b and genes involved in the regulation of RNA metabolic process. (XLSX $17 \mathrm{~kb}$ )

\section{Abbreviations}

3' UTR: 3' untranslated region; arnt/1a: Aryl hydrocarbon receptor nuclear translocator-like 1a; bhlhe41: Basic helix-loop-helix family, member e41; cirbp: Cold inducible RNA binding protein; DAVID: The Database for Annotation, Visualization and Integrated Discovery; Dpf: Days post fertilization; Dre: Danio rerio; F: Forward; GnRH: Gonadotropin releasing hormone; GO: Gene ontology; H2A: Histone 2A; hpf: h post fertilization; hpi: h post induction; KEGG: Kyoto Encyclopedia of Genes and Genomes; miRNAs: MicroRNAs; MO: Morpholino oligonucleotides; NOD: Oligomerization domain; Nts: Nucleotides; Per2: Period 2; qPCR: Quantitative polymerase chain reaction; R: Reverse; RIG-I: Retinoic acid-inducible gene 1; RPKM: Reads per kilo base per million; UTR: Untranslated region

\section{Acknowledgements}

We thank Taiwan Zebrafish core facility for providing technical guidance and plasmids. We thank Technology Commons, College of Life Science, Center for Systems Biology, National Taiwan University for excellent technical assistance with NGS and qPCR experiments. We thank the Taiwan Bioinformatics Core at the National Cheng Kung University, supported by the Taiwan Ministry of Science for assisting with computational analyses and data mining. We thank the Center for Genomic Medicine, National Cheng Kung University, for the support of NGS analyses. The authors would also like to express great appreciation to the staffs of the zebrafish Core at National Taiwan University for providing assistance in fish maintenance. We are also grateful to Dr. Shih-Kuo Chen for the helpful discussion in the regulation of circadian rhythm.

\section{Funding}

This work was supported by the Ministry of Science and Technology, Taiwan (101-2321-B-002-054-, 102-2121-B-002-041, 103-2321-B-002-009) and National Taiwan University (NTU NTU CESRP-10R70602A5 and NTU ERP-10R80600 to SJL

\section{Availability of data and materials}

No extra dataset except those presented in this article and the supporting information.

\section{Authors' contributions}

$\mathrm{ICH}$ carried out the clock gene functional studies, participated in the data analysis and drafted the manuscript. YCH carried out cold shock scheme analysis, miRNAome and transcriptome analysis and drafted the manuscript. HSS designed and supervised the next generation sequence experiments. TMC designed, analyzed and supervised miRNAome and transcriptome experiments, and edited the manuscript. SJL designed, analyzed, supervised all experiments, and revised the manuscript. All authors read and approved the final manuscript.

\section{Competing interests}

The authors declare that they have no competing interests.

\section{Consent for publication}

Not applicable.

\section{Ethics approval and consent to participate}

All experimental procedures on zebrafish were approved by the use of laboratory animal committee at National Taiwan University, Taipei, Taiwan (IACUC Approval ID: 100 Animal Use document No. 92) and carried out in accordance with the approved guidelines.

\section{Author details}

${ }^{1}$ Department of Life Science, National Taiwan University, Taipei 10617, Taiwan. ${ }^{2}$ Institute of Molecular Medicine, College of Medicine, National Cheng Kung University, Tainan 70456, Taiwan. ${ }^{3}$ Institute of Basic Medical Sciences, College of Medicine, National Cheng Kung University, Tainan 70456, Taiwan. ${ }^{4}$ Department and Graduate Institute of Aquaculture, National Kaohsiung Marine University, Kaohsiung 81157, Taiwan. ${ }^{5}$ Research Center for Developmental Biology and Regenerative Medicine, National Taiwan University, Taipei 10617, Taiwan. ${ }^{6}$ Center for Biotechnology, National Taiwan University, Taipei 10617, Taiwan. ${ }^{7}$ Center for Systems Biology, Taipei 10617, Taiwan.

Received: 17 May 2016 Accepted: 1 November 2016

Published online: 15 November 2016

\section{References}

1. Brett JR. Energetic responses of salmon to temperature. A study of some thermal relations in the physiology and freshwater ecology of sockeye salmon (Oncorhynchus nerka). Am Zool. 1971;11(1):99-113.

2. Donaldson MR, Cooke SJ, Patterson DA, Macdonald JS. Cold shock and fish. J Fish Biol. 2008;73(7):1491-530.

3. Mazeaud MM, Mazeaud F, Donaldson EM. Primary and secondary effects of stress in fish - some new data with a general review. T Am Fish Soc. 1977; 106(3):201-12.

4. Barton BA. Stress in fishes: a diversity of responses with particular reference to changes in circulating corticosteroids. Integr Comp Biol. 2002;42(3):517-25.

5. Barton BA. Physiological changes in fish from stress in aquaculture with emphasis on the response and effects of corticosteroids. 1991.

6. Flos R, Reig L, Torres P, Tort L. Primary and secondary stress responses to grading and hauling in rainbow-trout, salmo-gairdneri. Aquaculture. 1988; 71(1-2):99-106.

7. Chen WH, Sun LT, Tsai CL, Song YL, Chang CF. Cold-stress induced the modulation of catecholamines, cortisol, immunoglobulin $\mathrm{M}$, and leukocyte phagocytosis in tilapia. Gen Comp Endocrinol. 2002;126(1):90-100.

8. Mazeaud, MM and Mazeaud, F. Adrenergic responses to stress in fish. In: Pickering, AD, editor. Stress and fish. New York: Academic Press; 1981. p. $49-75$

9. Marcel Martinez-Porchas LRM-CRR-E. Cortisol and glucose: reliable indicators of fish stress? 2009.

10. Iwama GK, Vijayan MM, Forsyth RB, Ackerman PA. Heat shock proteins and physiological stress in fish. Am Zool. 1999;39(6):901-9.

11. Gracey AY. Interpreting physiological responses to environmental change through gene expression profiling. J Exp Biol. 2007;210(Pt 9):1584-92.

12. Teigen LE, Orczewska Jl, McLaughlin J, O'Brien KM. Cold acclimation increases levels of some heat shock protein and sirtuin isoforms in threespine stickleback. Comp Biochem Physiol A Mol Integr Physiol. 2015; 188:139-47. 
13. Windisch HS, Frickenhaus S, John U, Knust R, Portner HO, Lucassen M. Stress response or beneficial temperature acclimation: transcriptomic signatures in Antarctic fish (Pachycara brachycephalum). Mol Ecol. 2014;23(14):3469-82.

14. Ju Z, Dunham RA, Liu Z. Differential gene expression in the brain of channel catfish (Ictalurus punctatus) in response to cold acclimation. Mol Genet Genomics. 2002;268(1):87-95.

15. Long Y, Song G, Yan J, He X, Li Q, Cui Z. Transcriptomic characterization of cold acclimation in larval zebrafish. BMC Genomics. 2013;14:612.

16. Hori TS, Gamperl AK, Booman M, Nash GW, Rise ML. A moderate increase in ambient temperature modulates the Atlantic cod (Gadus morhua) spleen transcriptome response to intraperitoneal viral mimic injection. BMC Genomics. 2012;13:431.

17. Hassinen M, Paajanen V, Haverinen J, Eronen $H$, Vornanen M. Cloning and expression of cardiac Kir2.1 and Kir2.2 channels in thermally acclimated rainbow trout. Am J Physiol Regul Integr Comp Physiol. 2007;292(6):R2328-39

18. Deane EE, Woo NY. Cloning and characterization of the hsp70 multigene family from silver sea bream: modulated gene expression between warm and cold temperature acclimation. Biochem Biophys Res Commun. 2005; 330(3):776-83.

19. Ewart KV, Richards RC, Driedzic WR. Cloning of glycerol-3-phosphate dehydrogenase cDNAs from two fish species and effect of temperature on enzyme expression in rainbow smelt (Osmerus mordax). Comp Biochem Physiol B Biochem Mol Biol. 2001;128(3):401-12.

20. Spence R, Gerlach G, Lawrence C, Smith C. The behaviour and ecology of the zebrafish, Danio rerio. Biol Rev Camb Philos Soc. 2008;83(1):13-34.

21. Lim LP, Lau NC, Garrett-Engele P, Grimson A, Schelter JM, Castle J, Bartel DP, Linsley PS, Johnson JM. Microarray analysis shows that some microRNAs downregulate large numbers of target mRNAs. Nature. 2005; 433(7027):769-73.

22. Bartel DP. MicroRNAs: target recognition and regulatory functions. Cell. 2009;136(2):215-33.

23. Cross CE, Tolba MF, Rondelli CM, Xu M, Abdel-Rahman SZ. Oxidative stress alters miRNA and gene expression profiles in villous first trimester trophoblasts. Biomed Res Int. 2015;2015:257090.

24. Ci D, Song Y, Tian M, Zhang D. Methylation of miRNA genes in the response to temperature stress in Populus simonii. Front Plant Sci. 2015;6:921.

25. Chang TC, Wentzel EA, Kent OA, Ramachandran K, Mullendore M, Lee KH, Feldmann G, Yamakuchi M, Ferlito M, Lowenstein CJ, et al. Transactivation of miR-34a by p53 broadly influences gene expression and promotes apoptosis. Mol Cell. 2007;26(5):745-52.

26. Leung AK, Sharp PA. microRNAs: a safeguard against turmoil? Cell. 2007; 130(4):581-5.

27. Leung AK, Sharp PA. MicroRNA functions in stress responses. Mol Cell. 2010; 40(2):205-15.

28. Flynt AS, Thatcher EJ, Burkewitz K, Li N, Liu Y, Patton JG. miR-8 microRNAs regulate the response to osmotic stress in zebrafish embryos. J Cell Biol. 2009:185(1):115-27.

29. Yan B, Zhao LH, Guo JT, Zhao JL. miR-429 regulation of osmotic stress transcription factor 1 (OSTF1) in tilapia during osmotic stress. Biochem Biophys Res Commun. 2012;426(3):294-8.

30. Yang R, Dai Z, Chen S, Chen L. MicroRNA-mediated gene regulation plays a minor role in the transcriptomic plasticity of cold-acclimated zebrafish brain tissue. BMC Genomics. 2011:12:605.

31. Nielsen JA, Lau P, Maric D, Barker JL, Hudson LD. Integrating microRNA and mRNA expression profiles of neuronal progenitors to identify regulatory networks underlying the onset of cortical neurogenesis. BMC Neurosci. 2009;10:98.

32. Lukowski SW, Fish RJ, Martin-Levilain J, Gonelle-Gispert C, Buhler LH, Maechler P, Dermitzakis ET, Neerman-Arbez M. Integrated analysis of mRNA and miRNA expression in response to interleukin- 6 in hepatocytes. Genomics. 2015;106(2):107-15.

33. Okamura $\mathrm{H}$. Clock genes in cell clocks: roles, actions, and mysteries. J Biol Rhythms. 2004;19(5):388-99.

34. Yu W, Nomura M, Ikeda M. Interactivating feedback loops within the mammalian clock: BMAL1 is negatively autoregulated and upregulated by CRY1, CRY2, and PER2. Biochem Biophys Res Commun. 2002;290(3):933-41.

35. Rodriguez A, Griffiths-Jones S, Ashurst JL, Bradley A. Identification of mammalian microRNA host genes and transcription units. Genome Res. 2004;14(10A):1902-10.
36. Grimson A, Farh KK, Johnston WK, Garrett-Engele P, Lim LP, Bartel DP. MicroRNA targeting specificity in mammals: determinants beyond seed pairing. Mol Cell. 2007;27(1):91-105.

37. da Huang W, Sherman BT, Lempicki RA. Systematic and integrative analysis of large gene lists using DAVID bioinformatics resources. Nat Protoc. 2009; 4(1):44-57.

38. Long Y, Li L, Li Q, He X, Cui Z. Transcriptomic characterization of temperature stress responses in larval zebrafish. PLoS One. 2012;7(5): e37209.

39. Kanehisa M, Goto S, Furumichi M, Tanabe M, Hirakawa M. KEGG for representation and analysis of molecular networks involving diseases and drugs. Nucleic Acids Res. 2010;38(Database issue):D355-60.

40. Dunlap JC. Molecular bases for circadian clocks. Cell. 1999;96(2):271-90.

41. Honma S, Kawamoto T, Takagi Y, Fujimoto K, Sato F, Noshiro M, Kato Y, Honma K. Dec1 and Dec2 are regulators of the mammalian molecular clock. Nature. 2002;419(6909):841-4

42. Laposky AD, Bass J, Kohsaka A, Turek FW. Sleep and circadian rhythms: key components in the regulation of energy metabolism. FEBS Lett. 2008:582(1): 142-51.

43. Imaizumi T, Kay SA, Schroeder Jl. Circadian rhythms. Daily watch on metabolism. Science. 2007;318(5857):1730-1.

44. Wang Q, Tan X, Jiao S, You F, Zhang PJ. Analyzing cold tolerance mechanism in transgenic zebrafish (Danio rerio). PLoS One. 2014;9(7):e102492.

45. Scott GR, Johnston IA. Temperature during embryonic development has persistent effects on thermal acclimation capacity in zebrafish. Proc Nat Acad Sci U S A. 2012;109(35):14247-52.

46. Wang H, Zou Z, Wang S, Gong M. Global analysis of transcriptome responses and gene expression profiles to cold stress of Jatropha curcas $L$. PLoS One. 2013;8(12):e82817.

47. Chou MY, Hsiao CD, Chen SC, Chen IW, Liu ST, Hwang PP. Effects of hypothermia on gene expression in zebrafish gills: upregulation in differentiation and function of ionocytes as compensatory responses. J Exp Biol. 2008:211(Pt 19):3077-84.

48. Simon-Oppermann C, Hammel HT, Simon E. Hypothalamic temperature and osmoregulation in the Pekin duck. Pflugers Arch. 1979:378(3):213-21.

49. Shin HS, Bargiello TA, Clark BT, Jackson FR, Young MW. An unusual coding sequence from a Drosophila clock gene is conserved in vertebrates. Nature. 1985;317(6036):445-8

50. Ostberg $\mathrm{O}$. Circadian rhythms of food intake and oral temperature in "morning" and "evening" groups of individuals. Ergonomics. 1973; 16(2):203-9.

51. Lahiri K, Vallone D, Gondi SB, Santoriello C, Dickmeis T, Foulkes NS. Temperature regulates transcription in the zebrafish circadian clock. PLoS Biol. 2005;3(11):e351.

52. Zani F, Breasson L, Becattini B, Vukolic A, Montani JP, Albrecht U, Provenzani A, Ripperger JA, Solinas G. PER2 promotes glucose storage to liver glycogen during feeding and acute fasting by inducing Gys2 PTG and $G$ L expression. Mol Metab. 2013;2(3):292-305.

53. Kennaway DJ, Varcoe TJ, Voultsios A, Boden MJ. Global loss of bmal1 expression alters adipose tissue hormones, gene expression and glucose metabolism. PLoS One. 2013;8(6):e65255.

54. So AY, Bernal TU, Pillsbury ML, Yamamoto KR, Feldman BJ. Glucocorticoid regulation of the circadian clock modulates glucose homeostasis. Proc Natl Acad Sci U S A. 2009;106(41):17582-7.

55. Wendelaar Bonga SE. The stress response in fish. Physiol Rev. 1997;77(3): 591-625.

56. Grimaldi B, Bellet MM, Katada S, Astarita G, Hirayama J, Amin RH, Granneman JG, Piomelli D, Leff T, Sassone-Corsi P. PER2 controls lipid metabolism by direct regulation of PPARgamma. Cell Metab. 2010;12(5):509-20.

57. Kultz D. Molecular and evolutionary basis of the cellular stress response. Annu Rev Physiol. 2005:67:225-57. 